\section{Desigualdade educacional na ocorrência de obesidade abdominal por gênero e cor/raça: Estudo Pró-Saúde, 1999-2001 e 2011-2012}

\author{
Educational inequality in the occurrence of \\ abdominal obesity according to gender and \\ color/race: the Pro-Health Study, 1999-2001 \\ and 2011-2012
}

Desigualdad educacional en la ocurrencia de la obesidad abdominal por género y color/raza: Estudio Pro-Salud, 1999-2001 e 2011-2012

\section{Resumo}

Análise de dados seccionais do Estudo Pró-Saúde (1999-2001 e 2011 2012) revelou importante diferença de gênero e cor/raça na magnitude e variação temporal da desigualdade educacional na obesidade abdominal. Probabilidade de estar obeso foi gradativamente maior em mulheres (independentemente de cor/raça) e homens (pardos/pretos) menos escolarizados. Tais gradientes foram quantificados pelo indice relativo de desigualdade (IRD). Ao longo da década, observou-se redução da desigualdade em mulheres pardas/pretas ( $\triangle I R D: 0,5$; IC95\%: 0,2-1,1), subjacente ao relativo aumento da prevalência de obesidade abdominal entre as mais escolarizadas. Houve estabilidade do IRD em mulheres brancas e homens pardos/pretos, indicando crescimento similar da prevalência de obesidade abdominal nos subgrupos educacionais. Associação da escolaridade com a ocorrência de obesidade abdominal sofreu interação múltipla de fatores sociodemográficos. Nossos resultados encorajam a estratificação conjunta por gênero e cor/raça no estudo das desigualdades socioeconômicas na ocorrência da obesidade abdominal.

Obesidade Abdominal; Distribuição por Raça ou Etnia; Gênero e Saúde; Desigualdades em Saúde
Ronaldo Fernandes Santos Alves 1 Eduardo Faerstein 1 


\section{Introdução}

Em países de renda média e alta, a obesidade tem afetado principalmente mulheres em desvantagem social; no grupo dos homens, a relação entre posição socioeconômica e obesidade mostra-se inconsistente 1,2,3. No Brasil, mudanças socioeconômicas ocorridas nas últimas décadas implicaram o aumento da disponibilidade de alimentos ultraprocessados e redução da atividade física 4 . Observou-se tendência crescente da prevalência e incidência de obesidade associada à baixa posição socioeconômica entre mulheres 5,6.

As desigualdades socioeconômicas na ocorrência da obesidade, considerando interseção de gênero e cor/raça, assim como as respectivas variações temporais, são, ainda, inexplorados no país. Neste trabalho, estimamos magnitude e variação temporal da desigualdade educacional na ocorrência de obesidade abdominal nesses subgrupos demográficos.

\section{Métodos}

Estudo seccional com dados da linha de base (1999-2001) e fase 4 (2011-2012) do Estudo PróSaúde (EPS), programa de investigação longitudinal de funcionários técnico-administrativos de uma universidade no Rio de Janeiro ${ }^{7}$. Foram incluídos todos os funcionários do quadro efetivo da instituição que aceitaram participar do EPS, sendo excluídos gestantes e licenciados por motivos não relacionados à saúde.

Durante o trabalho de campo, houve $21,8 \%$ de não participação na linha de base $(\mathrm{N}=3.253)$, bem como $22,3 \%$ de não participação na fase 4 ( $N$ = 2.933). Em 1999-2001, 149 indivíduos não responderam as perguntas relativas às variáveis do estudo ou não tiveram aferida sua circunferência abdominal; outros 111 indivíduos foram excluídos da análise de dados por terem mais de 65 anos ou por terem autodeclarado cor/raça amarela ou indígena. Em 2011-2012, esses valores foram, respectivamente, 75 e 247. Obtivemos informação completa de 2.993 adultos em 19992001 (92\% dos participantes) e 2.611 adultos em 2011-2012 (89\% dos participantes).

Obesidade abdominal foi definida como circunferência - aferida em duplicata no nível umbilical - maior ou igual a $88 \mathrm{~cm}$ para mulheres e $102 \mathrm{~cm}$ para homens ${ }^{8}$. Questionário multidimensional, preenchido pelos próprios participantes, foi usado para avaliar escolaridade (fundamental completo; médio completo; superior completo), gênero (feminino; masculino), cor/raça (parda/ preta; branca) e idade (24 a 65 anos).
Análise dos dados abarcou prevalência com intervalo de 95\% de confiança (IC95\%) e teste de qui-quadrado de tendência em proporções. Aplicou-se o índice relativo de desigualdade (IRD) para estimar a magnitude da desigualdade educacional na ocorrência de obesidade abdominal. Esse índice é uma medida sumária da associação monotônica entre fator social politômico e desfecho de saúde, apropriada para comparações entre populações e temporalmente. Baseia-se em regressão log-linear ponderada, em que a variável dependente concerne ao desfecho de saúde e a variável independente remete a um escore numérico, definido a partir da frequência relativa acumulada dos subgrupos educacionais 9,10,11,12 . Nesta análise, o ordenamento partiu dos mais escolarizados. Variação temporal da estimativa de desigualdade entre os períodos de 1999-2001 e 2011-2012 foi analisada, criando-se uma variável (inquérito) correspondente às fases do EPS e incluindo-se um termo de interação (inquérito * escore numérico) no modelo de regressão. Todas as análises foram estratificadas por gênero e cor/ raça, sendo conduzidas no software R 3.1.2 (The R Foundation for Statistical Computing, Viena, Áustria; http://www.r-project.org).

Protocolos do EPS 1999-2001 e 2011-2012 foram aprovados pelos Comitês de Ética em Pesquisa do Hospital Universitário Pedro Ernesto e Instituto de Medicina Social (Universidade do Estado do Rio de Janeiro).

\section{Resultados}

Identificaram-se subgrupos de gênero e cor/raça mais escolarizados em 2011-2012 do que em 1999-2001 (Tabela 1). A maioria das mulheres e homens brancos concluiu, pelo menos, ensino superior completo, ao passo que a maioria das mulheres e homens pardos/pretos, no máximo, completou o ensino médio, em ambos os períodos estudados. Entre aqueles com diploma universitário, observamos duas vezes mais brancos que pardos/pretos em 1999-2001 e, aproximadamente, 1,6 vez em 2011-2012.

Prevalência de obesidade abdominal foi maior entre as mulheres em 1999-2001 e 20112012, especialmente entre as autodeclaradas pardas/pretas (Tabela 2). Entre os homens, não foram observadas diferenças de cor/raça estatisticamente significativas em ambos os períodos. Prevalência de obesidade abdominal passou de $35,1 \%$ (IC95\%: 33,4-36,9) para 54,5\% (IC95\% $52,6-56,5)$ na população do EPS, aumento verificado em todos os subgrupos demográficos.

Associação entre escolaridade e obesidade abdominal foi consistente e inversa entre 
Tabela 1

Tamanho populacional dos subgrupos educacionais por gênero e cor/raça. Estudo Pró-Saúde, 1999-2001 e $2011-2012$.

\begin{tabular}{|c|c|c|c|c|c|c|}
\hline \multirow[t]{2}{*}{ Variáveis } & \multicolumn{3}{|c|}{$1999-2001$ [n (\%)] } & \multicolumn{3}{|c|}{ 2011-2012 [n (\%)] } \\
\hline & Parda/Preta & Branca & Total & Parda/Preta & Branca & Total \\
\hline \multicolumn{7}{|l|}{ Mulheres } \\
\hline Fundamental completo & $225(28,1)$ & $85(9,7)$ & $310(18,5)$ & $97(13,1)$ & $30(4,0)$ & $127(8,5)$ \\
\hline Médio completo & $319(39,8)$ & $249(28,3)$ & $568(33,8)$ & $296(40,0)$ & $163(21,8)$ & $459(30,9)$ \\
\hline Superior completo & $257(32,1)$ & $544(62,0)$ & $801(47,7)$ & $347(46,9)$ & $554(74,2)$ & $901(60,6)$ \\
\hline Total & 801 & 878 & 1.679 & 740 & 747 & 1.487 \\
\hline \multicolumn{7}{|l|}{ Homens } \\
\hline Fundamental completo & $219(36,0)$ & $124(17,6)$ & $343(26,1)$ & $135(22,3)$ & $44(8,5)$ & $179(15,9)$ \\
\hline Médio completo & $256(42,0)$ & $238(33,8)$ & $494(37,6)$ & $260(42,9)$ & $184(35,5)$ & $444(39,5)$ \\
\hline Superior completo & $134(22,0)$ & $343(48,6)$ & $477(36,3)$ & $211(34,8)$ & $290(56,0)$ & $501(44,6)$ \\
\hline Total & 609 & 705 & 1.314 & 606 & 518 & 1.124 \\
\hline
\end{tabular}

Tabela 2

Prevalência de obesidade abdominal (\%) e intervalo de 95\% de confiança (IC95\%) por escolaridade, gênero e cor/raça; índice relativo de desigualdade (IRD) dos subgrupos demográficos. Estudo Pró-Saúde, 1999-2001 e 2011-2012.

\begin{tabular}{|c|c|c|c|c|c|c|}
\hline \multirow[t]{2}{*}{ Variáveis } & \multicolumn{3}{|c|}{ 1999-2001 [\% (IC95\%)] } & \multicolumn{3}{|c|}{ 2011-2012 [\% (IC95\%)] } \\
\hline & Parda/Preta & Branca & Total & Parda/Preta & Branca & Total \\
\hline \multicolumn{7}{|l|}{ Mulheres } \\
\hline Fundamental completo & $66,2(59,6-72,4)$ & $56,5(45,3-67,2)$ & $63,5(57,9-68,9)$ & $74,2(64,3-82,6)$ & $83,3(65,3-94,4)$ & $76,4(68,0-83,5)$ \\
\hline Médio completo & $48,3(42,7-53,9)$ & $45,8(39,5-52,2)$ & $47,2(43,0-51,4)$ & $77,0(71,8-81,7)$ & $73,0(65,5-79,6)$ & $75,6(71,4-79,5)$ \\
\hline Superior completo & $34,6(28,8-40,8)$ & $32,3(28,4-36,5)$ & $33,1(29,8-36,5)$ & $62,5(57,2-67,6)$ & $57,8(53,5-61,9)$ & $59,6(56,3-62,8)$ \\
\hline Total & $48,9(45,4-52,5)$ & $38,5(35,3-41,8)$ & $43,5(41,1-45,9)$ & $69,9(66,4-73,2)$ & $62,1(58,5-65,6)$ & $66,0(63,5-68,4)$ \\
\hline Valor de $p^{*}$ & $<0,001$ & $<0,001$ & $<0,001$ & $<0,001$ & $<0,001$ & $<0,001$ \\
\hline IRD (bruto) & $6,4(3,7-11,0)$ & $4,0(2,3-7,0)$ & $5,8(4,0-8,4)$ & $3,2(1,7-5,9)$ & $4,8(2,3-9,9)$ & $4,3(2,7-6,8)$ \\
\hline IRD (ajustado) ** & $3,1(1,7-5,5)$ & $2,5(1,4-4,5)$ & $3,1(2,1-4,6)$ & $1,7(0,9-3,5)$ & $3,8(1,8-8,0)$ & $2,9(1,8-4,7)$ \\
\hline \multicolumn{7}{|l|}{ Homens } \\
\hline Fundamental completo & $27,4(21,6-33,8)$ & $21,8(14,9-30,1)$ & $25,4(20,8-30,3)$ & $40,0(31,7-48,8)$ & $43,2(28,3-59,0)$ & $40,8(33,5-48,4)$ \\
\hline Médio completo & $23,0(18,0-28,7)$ & $30,2(24,5-36,5)$ & $26,5(22,7-30,6)$ & $41,5(35,4-47,8)$ & $42,4(35,1-49,9)$ & $41,9(37,2-46,6)$ \\
\hline Superior completo & $14,9(9,4-22,1)$ & $24,2(19,8-29,1)$ & $21,6(18,0-25,6)$ & $30,8(24,6-37,5)$ & $41,0(35,3-46,9)$ & $36,7(32,5-41,1)$ \\
\hline Total & $22,8(19,5-26,4)$ & $25,8(22,6-29,2)$ & $24,4(22,1-26,8)$ & $37,5(33,6-41,4)$ & $41,7(37,4-46,1)$ & $39,4(36,5-42,3)$ \\
\hline Valor de $p$ * & 0,008 & 0,951 & 0,170 & 0,049 & 0,723 & 0,179 \\
\hline IRD (bruto) & $2,6(1,3-5,3)$ & - & - & $1,9(1,0-3,5)$ & - & - \\
\hline IRD (ajustado) *夫 & $2,1(1,0-4,6)$ & - & - & $1,8(0,9-3,5)$ & - & - \\
\hline
\end{tabular}

* Valor de $p$ do teste de qui-quadrado de tendência em proporções;

** IRD ajustado por idade (variável contínua).

mulheres de ambas as categorias de cor/raça, em 1999-2001 e 2011-2012 (Tabela 2). Similarmente, tendência monotônica foi observada entre homens pardos/pretos, mas não entre homens brancos, nos dois momentos da pesquisa, indicando que cor/raça modificou o efeito da escolaridade na ocorrência de obesidade ab- dominal entre os homens. Deste modo, o IRD quantificou a probabilidade gradativamente maior de estar obeso entre mulheres (brancas e pardas/pretas) e homens (pardos/pretos) menos escolarizados.

Variação negativa do IRD ( $\triangle$ IRD: 0,5; IC95\%: $0,2-1,1)$ entre mulheres pardas/pretas indicou 
redução da desigualdade educacional na obesidade abdominal, subjacente ao crescimento relativamente maior da prevalência de obesidade abdominal entre as mais escolarizadas (Figura 1). Entre mulheres brancas e homens pardos/ pretos, observou-se estabilidade da medida de desigualdade, em virtude do aumento similar da prevalência de obesidade abdominal nos subgrupos educacionais.

\section{Discussão}

Prevalência de obesidade abdominal foi consistentemente maior entre mulheres, em particular entre as autodeclaradas pardas/pretas, não tendo sido encontrada diferença de cor/raça entre homens. Escolaridade esteve inversamente associada com obesidade abdominal entre mulheres de ambas as categorias de cor/raça e entre homens pardos/pretos, em 1999-2001 e 2011-2012. Observamos redução da desigualdade educacio- nal na obesidade abdominal entre mulheres pardas/pretas e estabilidade da desigualdade entre homens pardos/pretos e mulheres brancas.

Estudos recentes documentaram que a circunferência abdominal tem maior capacidade preditiva de desfechos relacionados à obesidade em comparação com outras avaliações antropométricas 8,13. Contudo, variação quanto ao ponto de corte pode influenciar estimativas de prevalência de obesidade abdominal; portanto, definimos obesidade abdominal conforme os parâmetros de risco muito elevado ${ }^{8}$.

Como limitações do estudo, assinalamos: população de base ocupacional que, embora contribua para validade interna, pode reduzir a generalização dos resultados para população geral; cerca de $20 \%$ dos elegíveis não participaram da linha de base e fase 4 , conquanto seja esperada intercambialidade entre população de estudo e população fonte; posição socioeconômica é um construto multidimensional, e nossa análise abordou apenas a escolaridade.

Figura 1

Logaritmo do índice relativo de desigualdade (IRD) ajustado por idade. Estudo Pró-Saúde (EPS), 1999-2001 e 2011-2012.

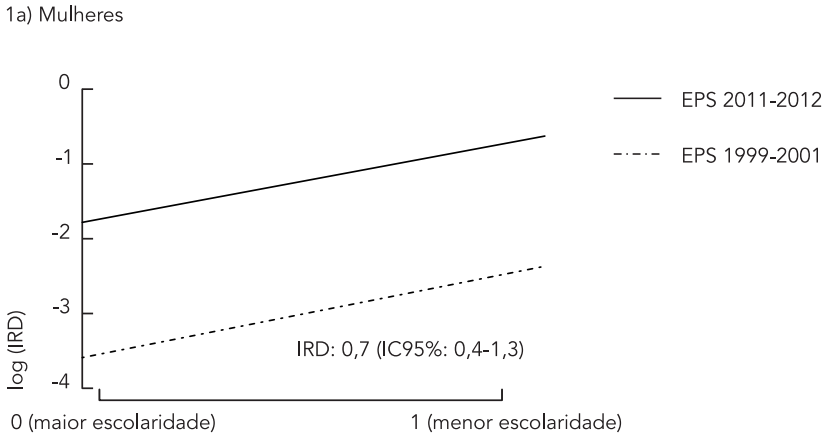

1c) Mulheres pardas/pretas

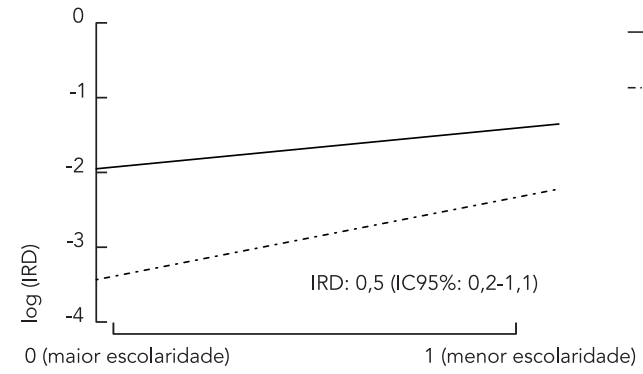

1b) Mulheres brancas

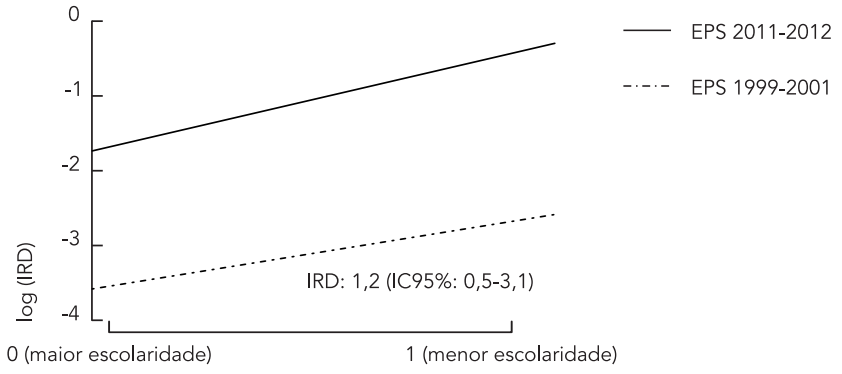

1d) Homens pardos/pretos

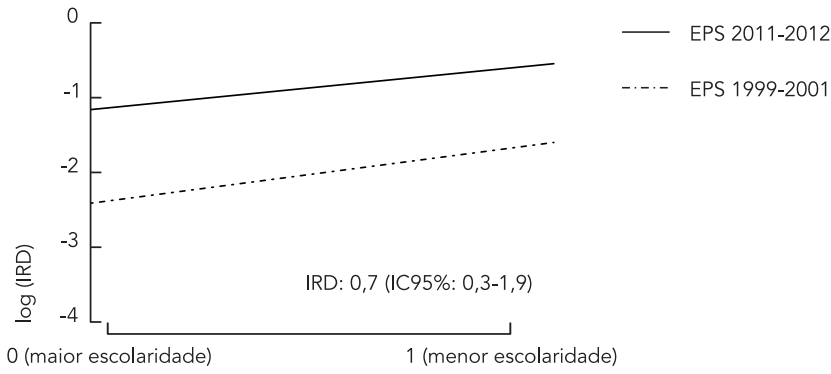


Evidências seccionais e longitudinais mostram padrão de associação inversa entre posição socioeconômica e obesidade, considerando-se principalmente escolaridade e o grupo de mulheres 1,2,3,5,6. Escolaridade influencia a obesidade diretamente por meio de modos e estilos de vida relacionados à alimentação, atividade física e outras práticas de saúde 14; indiretamente, por meio da ocupação e renda. Indivíduos de maior posição socioeconômica têm mais acesso à alimentação saudável e aos meios de redução voluntária do peso corporal. Por fim, fatores ambientais associados à posição socioeconômica também podem influenciar a obesidade 1,2,14

Poucos trabalhos enfocaram a complexa relação entre gênero, cor/raça, posição socioeconômica e obesidade 15,16,17,18,19. Nos Estados Unidos, encontraram-se diferenças na associação entre posição socioeconômica e obesidade, além de diferenças na tendência temporal da desigualdade socioeconômica na ocorrência de obesidade, segundo gênero e raça/etnicidade 15,16,17,18,19. Ferrer et al. 20 observaram redução da desigualdade educacional na ocorrência da obesidade entre mulheres mexicanas residentes de áreas urbanas, em face do aumento pronunciado da prevalência de obesidade entre as mais escolarizadas. Em nosso estudo, a associação da escolaridade com ocorrência de obesidade abdominal sofreu interação múltipla de fatores sociodemográficos. Apesar da melhoria do nível educacional em todos os subgrupos demográficos, observamos aumento da prevalência de obesidade abdominal, especialmente entre mulheres pardas/pretas com ensino superior completo. A validade de construto dos indicadores de posição socioeconômica, portanto, seu impacto, pode variar entre subgrupos de gênero e cor/ raça 21 . Por exemplo, experiências de discriminação racial podem estar relacionadas com excesso de peso entre mulheres pardas/pretas $22 \mathrm{de}$ maior escolaridade.

Nossos resultados encorajam a estratificação conjunta por gênero e cor/raça e o uso do IRD no estudo das desigualdades socioeconômicas na ocorrência da obesidade abdominal, bem como de outras desigualdades em saúde.

\section{Colaboradores}

R. F. S. Alves concebeu e planejou o presente estudo, analisou e interpretou os dados e elaborou a primeira versão do manuscrito. E. Faerstein participou da interpretação dos dados, revisão do conteúdo e redação do manuscrito. Ambos os autores aprovaram a versão final do texto.

\section{Agradecimentos}

Agradecemos aos participantes e à equipe do Estudo Pró-Saúde; ao Conselho Nacional de Desenvolvimento Científico e Tecnológico (CNPq) e à Fundação Carlos Chagas Filho de Amparo à Pesquisa do Estado do Rio de Janeiro (FAPERJ) pelo apoio financeiro. 


\section{Referências}

1. Dinsa GD, Goryakin Y, Fumagalli E, Suhrcke M. Obesity and socioeconomic status in developing countries: a systematic review. Obes Rev 2012; 13:1067-79.

2. McLaren L. Socioeconomic status and obesity. Epidemiol Rev 2007; 29:29-48.

3. Ball K, Crawford D. Socioeconomic status and weight change in adults: a review. Soc Sci Med 2005; 60:1987-2010.

4. Barreto SM, Pinheiro ARO, Sichieri R, Monteiro CA, Batista Filho M, Schmidt MI, et al. Análise da estratégia global para alimentação, atividade física e saúde, da Organização Mundial da Saúde. Epidemiol Serv Saúde 2005; 14:41-68.

5. Gigante DP, de França GVA, Sardinha LMV, Iser BPM, Meléndez GV. Variação temporal na prevalência do excesso de peso e obesidade em adultos: Brasil, 2006 a 2009. Rev Bras Epidemiol 2011; 14 Suppl 1:157-65.

6. Conde LC, Borges C. O risco de incidência e persistência da obesidade entre adultos brasileiros segundo seu estado nutricional ao final da adolescência. Rev Bras Epidemiol 2011; 14 Suppl 1:71-9.

7. Faerstein E, Chor D, Lopes CS, Werneck GL. Estudo Pró-Saúde: características gerais e aspectos metodológicos. Rev Bras Epidemiol 2005; 8:454-66.

8. World Health Organization. Waist circumference and waist-hip ratio: report of a WHO expert consultation. Geneva: World Health Organization; 2011.

9. World Health Organization. Handbook on health inequality monitoring with a special focus on low- and middle-income countries. Luxembourg: World Health Organization; 2013.

10. Harper S, Lynch J. Methods for measuring cancer disparities: using data relevant to health people 2010 cancer-related objectives. Bethesda: National Cancer Institute; 2005.

11. Alves RFS, Faerstein E. Desigualdade educacional na ocorrência de obesidade abdominal: Estudo Pró-Saúde. Rev Saúde Pública 2015; 49:65.

12. Moreno-Betancur M, Latouche A, Menvielle G, Kunst AE, Rey G. Relative Index of Inequality and Slope Index of Inequality: a structured regression framework for estimation. Epidemiology 2015; 26:518-27.
13. Tchernof A, Després JP. Pathophysiology of human visceral obesity: an update. Physiol Rev 2013; 93:359-404.

14. Sobal J. Obesity and socioeconomic status: a framework for examining relationships between physical and social variables. Med Anthropol 1991; 13:231-47.

15. Zhang Q, Wang Y. Socioeconomic inequality of obesity in the United States: do gender, age, and ethnicity matter? Soc Sci Med 2004; 58:1171-80.

16. Zhang Q, Wang Y. Trends in the association between obesity and socioeconomic status in US adults: 1971 to 2000. Obes Res 2004; 12:1622-32.

17. Wang Y, Beydoun MA. The obesity epidemic in the United States - gender, age, racial/ethnic, and geographic characteristics: a systematic review and meta-regression analysis. Epidemiol Rev 2007; 29:6-28.

18. Jackson CL, Szklo M, Yeh HC, Wang NY, Dray-Spira $\mathrm{R}$, Thorpe R, et al. Black-white disparities in overweight and obesity trends by educational attainment in the United States, 1997-2008. J Obes 2013; 2013:140743.

19. Ruopeng A. Educational disparity in obesity among US adults, 1984-2013. Ann Epidemiol 2015; 25:637-42.e5.

20. Ferrer CP, McMunn A, Dommarco JAR, Brunner EJ. Educational inequalities in obesity among Mexican women: time-trends from 1988 to 2012. PLoS One 2014; 9:e90195.

21. Ulijaszek SJ. Socio-economic status, forms of capital and obesity. J Gastrointest Canc 2012; 43:3-7.

22. Cunningham TJ, Berkman LF, Kawachi I, Jacobs DR, Seeman TE, Kiefe CI, et al. Changes in waist circumference and body mass index in the US CARDIA cohort: fixed-effect associations with selfreported experiences of racial/ethnic discrimination. J Biosoc Sci 2013; 45:267-78. 


\section{Abstract}

Cross-sectional data from the Pro-Health Study in 1999-2001 and 2011-2012 revealed important gender and color/race differences in the size and variation across time in educational inequalities related to abdominal obesity. Probability of obesity increased steadily in women (independently of color/race) and men (brown/black) with less schooling. These gradients were quantified according to the relative index of inequality (RII). Over the course of the decade, there was a reduction in inequality in brown/black women (ARII: 0.5; 95\%CI: 0.2-1.1), underlying a relatively higher increase in the prevalence of abdominal obesity in women with more schooling. RII was stable in white women and brown/black men, indicating a similar increase in the prevalence of abdominal obesity in educational subgroups. The association between schooling and abdominal obesity was affected by the multiple interaction of socio-demographic factors. Our results recommend joint stratification by gender and color/race in the study of socioeconomic inequalities related to abdominal obesity.

Abdominal Obesity; Race or Ethnic Group Distribution; Gender and Health; Health Inequalities

\section{Resumen}

Los análisis de datos seccionales del Estudio Pro-Salud durante los períodos 1999-2001 y 2011-2012 reveló una importante diferencia de género y color/raza en la magnitud y variación temporal de la desigualdad educacional en la obesidad abdominal. La probabilidad de estar obeso fue gradualmente mayor en mujeres (independiente de color/raza) y hombres (mulatos/ negros) menos escolarizados. Tales gradientes fueron cuantificados por el índice relativo de desigualdad (IRD). A lo largo de la década, se observó una reducción de la desigualdad en mujeres mulatas/negras (AIRD: 0,5; IC95\%: 0,2-1,1), subyacente al aumento relativamente mayor de la prevalencia de obesidad abdominal entre las más escolarizadas. Hubo estabilidad del IRD en mujeres blancas y hombres mulatos/negros, indicando crecimiento similar de la prevalencia de obesidad abdominal en los subgrupos educacionales. Asociación de la escolaridad con la ocurrencia de obesidad abdominal sufrió una interacción múltiple de factores sociodemográficos. Nuestros resultados muestran la estratificación conjunta por género y color/raza en el estudio de las desigualdades socioeconómicas en el ámbito de la obesidad abdominal.

Obesidad Abdominal; Distribución por Raza o Etnia; Género y Salud; Desigualdades en la Salud
Recebido em 14/Mai/2015

Versão final reapresentada em 03/Nov/2015

Aprovado em 10/Dez/2015 